\title{
LA ACTUACIÓN DEL PROFESIONAL DE LA PSICOLOGÍA EN LAS RESIDENCIAS DE PERSONAS MAYORES
}

\author{
Jaime Gutiérrez Rodríguez \\ Marta Florencia Abril Herrero \\ María Elvira Zorzo \\ María Ángeles García Torres \\ Estefanía González Arranz \\ Lorena María Sahagún Flores \\ Colegio Oficial de Psicología de Castilla y León \\ copcyl@cop.es
}

Fecha de Recepción: 2 Abril 2018

Fecha de Admisión: 10 Abril 2018

\section{RESUMEN}

El grupo de trabajo de Psicología del Envejecimiento del COPCyL considera importante contar con un profesional de la Psicología dentro de los equipos interdisciplinares de los centros residenciales de personas mayores para poder ofrecer una atención integral y de calidad. Con el objetivo de dar a conocer cuál es el papel de este profesional en las residencias se presenta un plan de actuación de las intervenciones centrándose, según a quiénes están dirigidas, en el/la residente (a nivel cognitivo, emocional y conductual), en la familia y en la institución. Pensamos que de esta manera queda justificada su actuación y la necesidad de su incorporación a los equipos de trabajo. Como línea de trabajo futura introducimos un instrumento para entrenar Marcos Relacionales en Mayores (MAREMA).

Palabras clave: papel del psicólogo-a; atención integral; residencias de personas mayores; teoría de los marcos relacionales

\section{ABSTRACT}

The psychology professinal s performance in residences for the elderly.

The COPCyL Aging Psychology Working Group believes is important to have a psychology professional within the interdisciplinary teams of the residential centers of elderly people in order to offer comprehensive care and quality. In order to make known what the role of this professional in the residences is presented a plan of action. It discusses their interventions by focusing, according to who they are directed, in the resident (cognitive, emotional and behavioral), in the family and in 


\section{LA ACTUACIÓN DEL PROFESIONAL DE LA PSICOLOGÍA EN LAS RESIDENCIAS DE PERSONAS MAYORES}

the institution. We think that in this way their performance and the need for their incorporation to work teams is justified. As a future line of work we introduce an instrument to train relational frames in older (MAREMA).

Key Words: psycologist role; comprehensive care; residences for the elderly; relational frames theory (RFT)

\section{LA ACTUACIÓN DEL PROFESIONAL DE LA PSICOLOGÍA EN LAS RESIDENCIAS DE PERSONAS MAYORES}

Es labor del Colegio Oficial de Psicología de Castilla y León (COPCyL) divulgar cuáles son las funciones del profesional de la Psicología y en qué consiste su intervención. De ahí que se presente este documento elaborado por el Grupo de Trabajo de Psicología del Envejecimiento del COPCyL. Con el objetivo de aproximar a las administraciones e instituciones públicas y/o privadas una descripción del plan de trabajo y actuación del psicólogo-a justificando la necesidad de un profesional de la Psicología en las residencias de mayores.

\section{JUSTIFICACIÓN}

El Colegio Oficial de Psicólogos de España en el año 1998 ya establece que dentro del marco de la intervención social tiene su ámbito de actuación el profesional de la Psicología que trabaja en residencias geriátricas.

En cuanto a la contratación de psicólogos-as en esta área, existe una tendencia a ir en aumento en el sector público y aún más en el sector privado. No obstante, queremos poner de manifiesto que esta contratación es insuficiente porque no todos los centros residenciales de personas mayores cuentan con un psicólogo-a en su plantilla de trabajadores y la figura del psicólogo debe estar presente en el equipo interdisciplinar si se desea prestar una atención integral y centrada en la persona.

A pesar de que aún no existe en nuestro país una normativa que regule la actividad del psicólogo-a en ese ámbito, tanto la normativa autonómica (Ley 5/2003, de 3 de abril de Atención y Protección a las Personas Mayores de Castilla y León) como la normativa estatal (Ley 39/2006, de 14 de diciembre de Promoción de la Autonomía Personal y Atención a las personas en situación de dependencia) reconocen el derecho de la persona mayor dependiente a recibir una atención integral.

Esta atención integral requiere de una intervención biopsicosocial, por lo que no podemos obviar el lado emocional de estas personas, sus aficiones e intereses, su personalidad, su autoestima, sus miedos, inquietudes o preocupaciones, su autoconcepto, su inteligencia, su memoria, sus relaciones sociales, su satisfacción, su calidad de vida...

En el colectivo de personas mayores se dan no solo patologías orgánicas sino psíquicas* y sociales y cada una de ellas debe estar atendida por especialistas para abordarse de una manera pertinente.

La persona responsable de la toma de decisiones para derivar a otro centro a ciertos residentes con limitaciones psíquicas o de evaluar el estado cognitivo y afectivo no puede delegarse en un profesional que no está cualificado para estas competencias. El psicólogo-a también es clave como agente facilitador durante la adaptación a la nueva situación. Tanto para los mayores como para sus familiares, es el profesional indicado para desarrollar técnicas cognitivas, entrenamiento en habilidades sociales (tanto para los mayores como para el personal y cuidadores informales), establecer pautas de modificación de conductas para residentes considerando sus dificultades, su grado de autonomía, coordinación y autodeterminación.

*En España, en el informe del Instituto de Mayores y Servicios Sociales (IMSERSO 2005), se contabiliza, en personas institucionalizadas, una prevalencia de un $12,4 \%$ personas con rasgos de depresión mayor y un 30,5 \% que presentan sintomatología depresiva., 
A continuación presentamos un plan de actuación para el psicólogo-a de residencias de personas mayores. En el que se detallan sus ámbitos de intervención, dirigidas hacia residentes (a nivel cognitivo, emocional y conductual), a la familia y a la institución. Para finalizar, incluimos unas líneas futuras de actuación donde se presenta un instrumento de trabajo basado en la Teoría de Marcos Relacionales.

\section{INTERVENCIÓN DIRIGIDA A RESIDENTES}

Los objetivos generales que persigue el psicólogo-a con sus intervenciones son:

Promover la salud, el bienestar, la autonomía y la calidad de vida de los/las residentes

Mejorar el estado funcional de la persona y desarrollar sus competencias

Potenciar la adaptación e integración en el centro

Mejorar las funciones cognoscitivas preservadas y/o residuales con el fin de enlentecer el deterioro cognitivo y restaurar las habilidades cognitivas en desuso partiendo de las funciones intelectuales conservadas.

Favorecer las competencias cognitivas y emocionales para facilitar el afrontamiento de problemas.

Prevenir el desarrollo de problemas emocionales, ansiedad, depresión...

Promover la activación conductual del residente, cómo parte preventiva de trastornos depresivos así como orientación hacia valores y metas en la época de vida en la que se encuentran.

Orientar la activación conductual de forma individual siendo coherente con su escala de valores, para evitar aislamiento y romper patrones de evitación experiencial.

Promover la conducta como motor, para generar pensamientos y emociones agradables.

Mejorar la atención global del/de la residente

Potenciar el trabajo interdisciplinar y la visión biopsicosocial.

\section{INTERVENCIÓN DEL PROFESIONAL DE LA PSICOLOGÍA EN EL ÁREA COGNITIVA}

Es fundamental la valoración inicial por parte del psicólogo-a cuando el usuario llega al centro. En función de la misma se fijan los objetivos a trabajar en cada área cognitiva y se elaboran programas de psicoestimulación adaptados a cada caso. En general, es habitual que se trabaje la activación de las siguientes funciones:

Tabla 1. Objetivos específicos a trabajar en las principales funciones psicológicas

\begin{tabular}{|c|c|}
\hline $\begin{array}{l}\text { FUNCIONES } \\
\text { PSICOLÓGICAS }\end{array}$ & OBJETIVOS ESPECÍFICOS \\
\hline ORIENTACIÓN & $\begin{array}{l}\text { - Favorecer y activar la orientación espacial y temporal para mantenerla el mayor tiempo } \\
\text { posible. } \\
\text { - Mantener la orientación de la persona. } \\
\text { - Activar la memoria personal o autobiográfica. }\end{array}$ \\
\hline MEMORIA & $\begin{array}{l}\text { - Ejercitar los diferentes tipos y procesos de la memoria. } \\
\text { - Ejercitar la memoria inmediata, mediante la presentación de estímulos visuales y } \\
\text { auditivos. } \\
\text { - Optimizar la memoria reciente, mediante la presentación de estímulos verbales y } \\
\text { visuales. } \\
\text { - Ofrecer elementos emotivos que favorezcan la permanencia de los recuerdos lejanos. } \\
\text { - Potenciar la orientación de la persona. } \\
\text { - Conservar la memoria autobiográfica. } \\
\text { - Ejercitar la memoria fotográfica. }\end{array}$ \\
\hline
\end{tabular}




\begin{tabular}{|c|c|}
\hline & - Potenciar la capacidad de evocar recuerdos difusos. \\
\hline LENGUAJE & $\begin{array}{l}\text { - Maximizar las capacidades lingüísticas preservadas. } \\
\text { - Ejercitar el lenguaje automático. } \\
\text { - Optimizar la expresión verbal. } \\
\text { - Mantener y estimular la comprensión verbal. } \\
\text { - Potenciar la capacidad de repetición. } \\
\text { - Favorecer la incitación y la fluidez verbal. } \\
\text { - Ejercitar la mecánica de la lecto-escritura. } \\
\text { - Mejorar la expresión escrita. } \\
\text { - Ejercitar la comprensión verbal y escrita. } \\
\text { - Favorecer la comprensión y expresión del lenguaje no escrito. }\end{array}$ \\
\hline CÁLCULO & $\begin{array}{l}\text { - Favorecer la capacidad de concentración. } \\
\text { - Mantener el concepto de número. } \\
\text { - Ejercitar habilidades de conteo. } \\
\text { - Ejercitar habilidades de cálculo simple. } \\
\text { - Ejercitar habilidades aritméticas. } \\
\text { - Ejercitar la función ejecutiva a través del cálculo: razonamiento y resolución de } \\
\text { problemas } \\
\text { - Mantener el concepto y uso del dinero. }\end{array}$ \\
\hline PRAXIAS & $\begin{array}{l}\text { - Favorecer las habilidades viso-constructivas. } \\
\text { - Optimizar la motricidad fina como requisito previo para la motricidad con } \\
\text { intencionalidad. } \\
\text { - Favorecer la producción de actos motores voluntarios. } \\
\text { - Mantener la mecánica de la escritura como ejercicio de las praxias gráficas. }\end{array}$ \\
\hline GNOSIAS & $\begin{array}{l}\text { - Mejorar la adquisición de habilidades de reconocimiento y percepción de elementos } \\
\text { visuales, auditivos, táctiles y olfativo y su significado y su interpretación. }\end{array}$ \\
\hline EJECUTIVAS & $\begin{array}{l}\text { - Favorecer la reversibilidad y flexibilidad cognitiva. } \\
\text { - Favorecer la capacidad de concentración. } \\
\text { - Ejercitar capacidad de planificación. } \\
\text { - Ejercitar capacidad de secuenciación e inhibición de conductas inadecuadas. } \\
\text { - Potenciar la capacidad de solucionar un problema }\end{array}$ \\
\hline
\end{tabular}

Fuente: Basado en el documento http://www.afatorrevieja.es/pdf/programas.pdf

Pero también, en cuanto a intervención a nivel cognitivo, pensamos que hay que considerar:

\section{Tabla 2. Otras intervenciones}

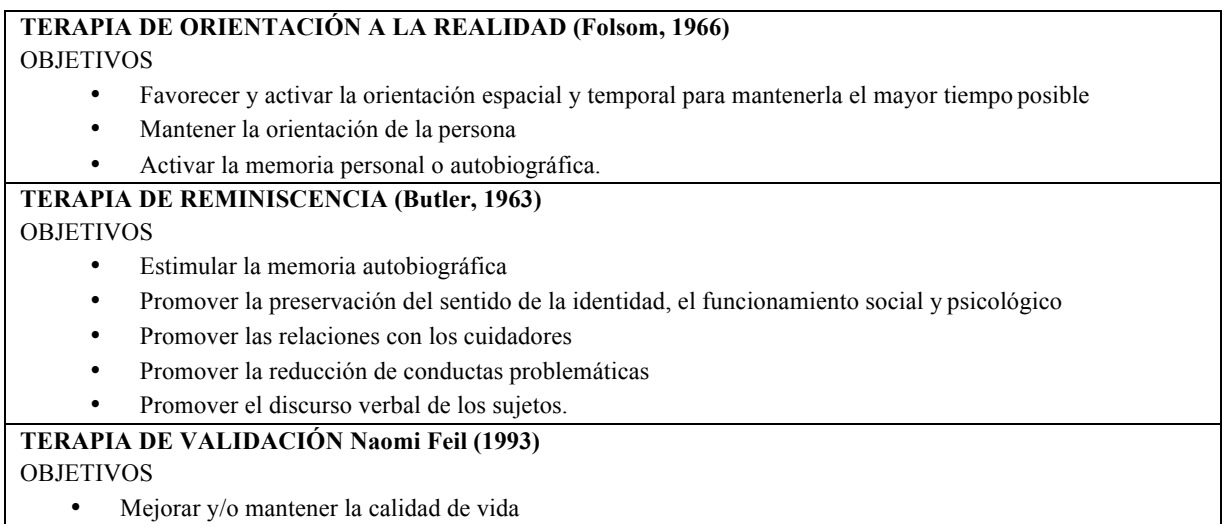


- Satisfacer las necesidades que presenta el usuario-a

TERAPIA MULTISENSORIAL. ENFOQUE "Snoozelen"

OBJETIVOS

- Mejorar la asimilación de la información sensorial que se les ofrece, optimizando su relación con el entorno y sus aprendizajes.

- Mejorar la calidad de vida

\section{INTERVENCIÓN DEL PROFESIONAL DE LA PSICOLOGÍA EN EL ÁREA EMOCIONAL}

El papel del psicólogo-a es fundamental a la hora de trabajar las emociones en las personas mayores porque estas nos acompañan en nuestra vida, porque vivir es sentir y porque las emociones son fundamentales para la adaptación. Además, estas están íntimamente ligadas a la salud.

\section{OBJETIVOS ESPECÍFICOS:}

Adquirir un mayor conocimiento de las propias emociones derivadas de la adaptación al proceso de envejecimiento.

Conocer y aplicar nuevas estrategias de regulación emocional.

Conocer con mayor profundidad la relación entre las emociones y los pensamientos y otros procesos cognitivos.

Reconocer y comprender los estados emocionales propios y de los demás y los procesos que Ios conforman: Comprensión emocional

Conocer y aplicar nuevas estrategias de regulación emocional, incluyendo el desarrollo de habilidades para generar emociones positivas y de habilidades para automotivarse.

Tomar conciencia y desarrollar un mayor nivel de autoestima en la nueva imagen de persona mayor.

Desarrollar un estilo asertivo para una mejora de las relaciones sociales tanto con la familia, compañeros y personal de atención directa.

Adquirir nuevas estrategias para la toma de decisiones con respecto a la ocupación del tiempo y la asunción de los errores.

Los programas de educación emocional (Ej. EMOCIONA T) centran su intervención en la adquisición de competencias básicas para afrontar situaciones que la vida plantea y a su vez, fomentan el desarrollo integral del individuo.

\section{INTERVENCIÓN DEL PROFESIONAL DE LA PSICOLOGÍA A NIVEL CONDUCTUAL}

El psicólogo-a interviene a nivel conductual, a través de la evaluación, la activación conductual y el análisis de la historia de vida de cada residente, teniendo en cuenta sus gustos, intereses y la orientación de valores personales.

La activación conductual es básica para la prevención de trastornos depresivos. Cabe destacar que la prevalencia del trastorno depresivo en personas mayores llega hasta el 25\% (Blazer, 2002; Reynolds y Kupfer, 1999), incrementándose de forma alarmante en aquellos institucionalizados (Jongenelis Pot, Eisses, Beekman, Kluiter y Ribbe, 2004).

\section{OBJETIVOS ESPECÍFICOS}

Evaluar la historia de vida de cada residente, realizando análisis funcional de su conducta.

Asignar los programas en función de sus intereses y motivaciones. (programas terapia ocupacional, voluntariado, laborterapia, musicoterapia, etc.).

Fomentar sentimiento de utilidad y valía personal en cada uno de los programas, a través de sus aportaciones y participación. 


\section{LA ACTUACIÓN DEL PROFESIONAL DE LA PSICOLOGíA EN LAS RESIDENCIAS DE PERSONAS MAYORES}

Establecer objetivos y metas dentro del entorno residencial. Análisis de la conducta manifiesta.

\section{PROGRAMA DE BIENVENIDA Y ACOGIDA AL CENTRO RESIDENCIAL}

Esta fase es muy importante. En ella se va a realizar una entrevista individual tanto con familiares como con residentes. El residente llega al centro residencial cargado de eventos privados, ideas prefijadas al respecto y anticipaciones de cómo va ser su vida a partir de ahora, por ello es prioritario que el profesional de la Psicología se encargue del recibimiento, con el fin de manejar, validar y ayudar en el afrontamiento de los eventos privados que surjan.

\section{EVALUACIÓN INICIAL - MODELO HISTORIA DE VIDA}

En esta fase se elabora la revisión de la historia de vida del residente y análisis de motivaciones e intereses. Es positivo, plantear que la adaptación al centro es progresiva, y que la persona decide el ritmo en la evolución. Siguiendo esta línea, hay que indagar en sus expectativas en el centro residencial, lo que quiere conseguir con su ingreso en el centro y cómo le gustaría vivir a partir de ahora.

El psicólogo-a realiza una evaluación para conocer el perfil cognitivo del usuario-a. Es decir, qué procesos cognitivos tiene alterados y qué áreas tiene preservadas. Además, hay que considerar que un objetivo secundario de la evaluación cognitiva es establecer una línea base de funcionamiento cognitivo que pueda ser usada para determinar la eficacia de las intervenciones.

\section{ASIGNACIÓN PROGRAMA ACTIVIDADES - ELABORACIÓN P.A.I}

Teniendo en cuenta la información recogida en el modelo de historia de vida, el psicólogo-a junto al equipo interdisciplinar realiza el Programa de Atención Individualizada (PAI). Se revisa el programa con el residente, con el fin de fomentar su autonomía y autodeterminación, resaltando la importancia de la adherencia al PAI desde nuestro punto de vista profesional, reforzando sus beneficios. EI PAI debe ser revisado de forma periódica, aproximadamente cada tres meses o de forma excepcional ante cambios que hagan necesaria dicha revisión.

El siguiente paso dentro del área cognitiva consiste en diseñar los programas de psicoestimulación que resulten más adecuados para desarrollar una dinámica grupal pero ajustándolos a las características del individuo (en función de los resultados de su evaluación y contemplando otros aspectos emocionales, conductuales y sociales que puedan estar comprometiendo su funcionamiento). De ahí la importancia de la especialización ya que una intervención cognitiva poco ajustada a los problemas y estadio evolutivo del/la residente puede crear frustración y estrés, y por tanto, deteriorar el curso evolutivo de la enfermedad.

\section{APOYO E INTERVENCIÓN INDIVIDUAL. REVISIÓN DE OBJETIVOS Y METAS. PLANIFICACIÓN DE CONDUCTAS.}

Cuando una persona mayor es institucionalizada, se adapta mejor si se involucra dentro del programa de actividades del centro residencial, aunque en muchas ocasiones la persona se encuentra inmersa en una programación que no tiene nada que ver con su repertorio de intereses anteriores, mostrando resistencia ante las actividades, respondiendo con evitación e inactividad, lo cual supone una disminución progresiva de reforzadores positivos.

La persona mayor tiene que adaptarse continuamente a cambios, ya sean físicos, sociales o psíquicos y también a la elaboración de pérdidas y procesos de duelo. El profesional de la Psicología acompaña al residente a través de los cambios, los prepara para afrontarlos y prevenir y/o tratar posibles patologías que se deriven.

La intervención psicológica, a nivel emocional, es fundamental ya que la institucionalización 
conlleva pérdidas funcionales, psíquicas y/o sensoriales, por lo que el apoyo psicológico puede prevenir la depresión y la ansiedad, en esta etapa de la vida es necesario intervenir para una mejor adaptación al centro residencial, ya que los sentimientos más frecuentes a los que se enfrentan los/las residentes son los de inutilidad, disconformidad e impotencia.

El psicologo-a, realiza un análisis funcional del patrón de conducta de cada residente, imprescindible para trabajar a nivel emocional y conductual. Se acuerda con el/la residente una programación de conductas a llevar a cabo, manteniendo aquellas que para la persona han sido y son refuerzos en su vida y añadiendo comportamientos nuevos que puede realizar en el centro. Se establecen de forma operativa, de esa manera se puede registrar la evolución.

El psicólogo-a va a realizar un seguimiento para poder realizar modificaciones en el programa según se vayan produciendo cambios y se establezcan nuevos objetivos de intervención que quedarán reflejados en las reuniones periódicas del equipo multidisciplinar (en el PAI).

Tabla 4. Programación individualizada de actividades

\begin{tabular}{|l|}
\hline Patrón de conductas positivas y saludables instauradas previamente en la historia de vida del residente. \\
\hline Conductas a llevar a cabo en el centro, dentro de su repertorio de intereses y aficiones. \\
\hline Participación programa de actividades del centro (Actividades asignadas). \\
\hline Adherencia a la rutina diaria del centro. \\
\hline
\end{tabular}

Se debe respetar el tiempo sin actividad o a la decisión de no ser partícipe. Destacando que en ocasiones la no actividad conductual, no supone que el residente esté inactivo.

\section{INTERVENCIÓN DEL PROFESIONAL DE LA PSICOLOGÍA CON FAMILIAS}

Cuando una persona accede a un centro residencial, no ingresa de forma aislada, ni comienza una nueva historia. Es una persona que sigue siendo miembro de una familia determinada y su historia personal continúa, ingresa con un pasado y tiene un nuevo futuro.

La familia con el modelo de atención centrada en la persona se establece como un pilar fundamental en la atención al residente, nos ayuda a conocer su historia, aficiones, enfermedades, etc. Su grado de implicación, su capacidad para afrontar la situación y su madurez a la hora de tomar decisiones en el caso que el residente no pueda tomarlas, es clave.

El programa de intervención con familias se realiza de forma individual y grupal.

De manera individual se centra en la atención y apoyo al proceso de adaptación de la propia familia en todas las fases que concurren en una residencia:

-Preingreso: Indecisión, conflictos familiares por la toma de decisión, ideas estereotipadas sobre el ingreso en un centro.

-Ingreso: Sentimientos de culpa-tristeza, depresión, sentimiento de vacío.

-Adaptación: Desconfianza del centro, dudas sobre el tipo de atención que recibe su familiar, necesidad de justificación, excesiva dedicación, conflictos con el personal.

-Integración: clarificación del rol como familiar, participación en el centro y en la vida del usuario-a, plantear sugerencias.

-Etapa final: dificultad en la toma de decisiones difíciles, acompañamiento final.

De forma grupal, la intervención va a ser con grupos psicoeducativos donde se aprenden estrategias para comunicarse con el residente y estrategias de afrontamiento y resolución de problemas, también se pueden programar actividades intergeneracionales. 


\section{LA ACTUACIÓN DEL PROFESIONAL dE LA PSICOLOGía EN LAS RESIDENCIAS DE PERSONAS MAYORES}

\section{OBJETIVOS ESPECÍFICOS:}

Dar apoyo psicológico centrado fundamentalmente en la desculpabilización.

Sensibilizar sobre la importancia del papel de la familia como soporte afectivo, cooperador en los cuidados y el vínculo con la continuidad de vida y las redes sociales de la comunidad.

Orientar para que la familia sepa cómo apoyar la adaptación de la persona mayor al centro.

Intervenir precozmente ante los posibles conflictos que puedan ocurrir con el personal de la residencia.

Afianzar la confianza de la familia en los profesionales del centro, reduciendo su inseguridad y temores sobre cómo es atendida la persona.

Intensificar la participación de las familias en el plan de vida y de cuidados de la persona en situación de dependencia.

Formar a través de sesiones grupales sobre gestión de estrés y gestión emocional

Apoyar psicológicamente a familias ante un empeoramiento de salud de su familiar e inminente fallecimiento 0 enfermedad terminal.

Acompañar en el duelo.

\section{INTERVENCIÓN DEL PROFESIONAL DE LA PSICOLOGÍA EN LA INSTITUCIÓN}

En la última normativa Modelo de Atención Integral Centrada en la persona, establecida por la Gerencia de Servicios Sociales, se parte de la premisa de realizar un Modelo de Historia de vida de cada residente con el fin de conocer, validar y posicionar a la persona en un contexto valioso, como es su experiencia vital, que debe ser llevado a cabo por el personal de referencia que tenga cada residente. Consideramos que esto contribuye a la creación de un contexto de referencia saludable, siempre que sea guiado y coordinado por el profesional de la Psicología. También esto es compatible con la intervención del psicólogo tanto en su elaboración como en el proceso de elección de la persona de referencia del residente.

Los profesionales que trabajan dentro del ámbito sanitario deben comprender la visión biopsicosocial y asimilarla de tal forma que sea una premisa que se ponga en práctica dentro de su campo de actuación profesional. Cuando coordinamos equipos y formamos profesionales los/as psicólogos/as tenemos que incidir en la relevancia de este enfoque para poder conseguir trabajar siempre por la mejora de la salud y la calidad de vida del residente. De esta forma, damos valor a cada una de las áreas que conforman al usuario-a en su totalidad y al mismo tiempo, visibilizamos la importancia de los distintos profesionales que trabajan dentro del centro por y para los residentes.

La coordinación y la formación de equipos dentro de los centros residenciales es una de las funciones fundamentales del psicologo-a para dar fuerza a este enfoque, y por lo tanto, para que los centros se conviertan en centros de atención sociosanitaria donde no se trabajan con enfermedades sino con personas y siempre a través de equipos interdisciplinares.

El profesional de la Psicología cuenta con un amplio campo de intervención: clínica, educativa, recursos humanos, investigación, intervención social (Mielgo, Ortiz y Ramos, 2001). Lo que le aporta un enfoque que incide de forma positiva sobre el nivel de calidad de la atención a las personas mayores. Ya que a la hora de formar y coordinar equipos, sus conocimientos especializados en geriatría y su vocación hacia un trabajo interdisciplinar hacen que sea un profesional cualificado en áreas cognitivas, funcionales, sociales, psicoafectivas, en la evaluación de las mismas y en la elaboración de programas de intervención.

En este apartado nos vamos a centrar en el asesoramiento, la coordinación y la formación, siendo considerados programas de atención indirecta porque no trabajamos directamente con el residente, sino con su contexto. 
Los profesionales de la Psicología especializados en esta área pueden asesorar y resolver dudas al personal y a la dirección del centro sobre la puesta en marcha de programas y las necesidades que existen tanto a nivel de usuarios-as como a nivel de profesionales. Uno de los programas que consideramos que debemos potenciar es la elaboración de la Historia de vida junto con el profesional de referencia. El/la psicólogo/a debe motivar y asesorar sobre las pautas a seguir en el proceso.

Por su parte, en la coordinación y gestión de equipos, se trabaja para favorecer la cohesión grupal, la adaptación y el afrontamiento de situaciones que pueden ser complicadas en el día a día, la motivación de todos los miembros del equipo, la comunicación eficaz, la toma de decisiones de forma conjunta y la coordinación entre profesionales.

El psicólogo/a, se encarga de impartir actividades formativas dirigidas tanto a los profesionales que forman parte del centro para ayudarles a entender y gestionar las distintas situaciones a las que se enfrentan.

\section{OBJETIVOS ESPECÍFICOS:}

Comprender la relevancia de todos los profesionales del centro en la atención y tratamiento.

Desarrollar la Historia de vida del/de la residente con su profesional de referencia.

Aprender a hacer adaptaciones en el centro para mejorar la calidad de vida del/de la residente.

Trabajar la comunicación y habilidades comunicativas entre profesionales y entre profesional residente, profesional - familia.

Aprender a gestionar conflictos.

Proporcionar las bases para una toma de decisiones eficaz.

Prevenir el maltrato psicológico en el centro residencial.

Prevenir el burnout.

Tabla 5. Programas de intervención en la institución

\begin{tabular}{l}
\hline ATENCIÓN INTEGRAL AL/LA RESIDENTE \\
Objetivos: \\
Definir las necesidades de los/las residentes usando un enfoque biopsicosocial. \\
Detallar los términos de bienestar y calidad de vida. \\
Aprender a elaborar la Historia de vida del/la residente y la relevancia del mismo como herramienta de trabajo. \\
Identificar la responsabilidad de los distintos profesionales en la salud del/la residente. \\
Identificar qué es maltrato psicológico dentro de un centro residencial y trabajar para prevenirlo. \\
\hline LA COMUNICACIÓN CON EL/LA RESIDENTE. IMPORTANCIA SOBRE LA SALUD \\
Objetivos: \\
Establecer qué engloba la comunicación. \\
Discriminar los tipos de comunicación existentes y su relevancia en el proceso comunicativo. \\
Analizar la importancia de una buena comunicación entre profesionales como base de un tratamiento eficaz. \\
Trabajar las habilidades comunicativas interpersonales. Asertividad con el/la residente, las familias de los residentes y los \\
compañeros de trabajo. \\
Preparar a los profesionales para transmitir malas noticias. \\
\hline EMPATÍA Y COMPRENSIÓN DEL RESIDENTE \\
Objetivos: \\
Identificar la empatía. \\
Fundamentar los elementos de la empatía. \\
Trabajar mediante el uso de dinámicas y casos la relevancia de la empatía en el tratamiento de residentes. \\
\hline DEONTOLOGÍA PROFESIONAL. NUESTRA RESPONSABILIDAD \\
Objetivos: \\
Comprender qué es la deontología profesional. \\
Analizar distintos casos en los que no se cumple con la deontología profesional dentro del centro. \\
\hline
\end{tabular}




\section{LA ACTUACIÓN DEL PROFESIONAL DE LA PSICOLOGíA EN LAS RESIDENCIAS DE PERSONAS MAYORES}

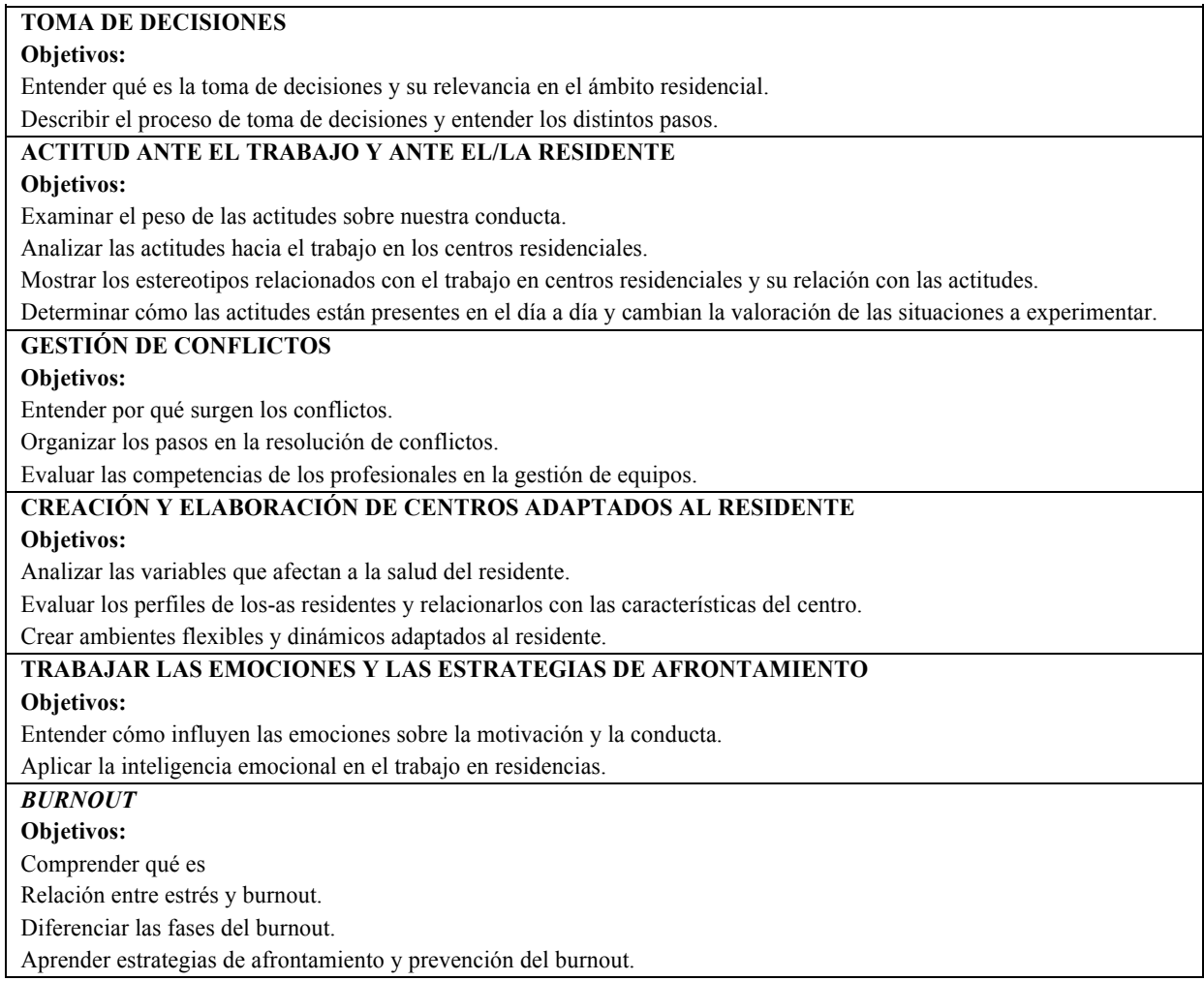

\section{LÍNEAS FUTURAS DE INTERVENCIÓN}

MAREMA (Instrumento para el trabajo de Marcos Relaciones en Mayores)

Los conceptos y su imagen mental respecto a la relación que establecemos entre la palabra, el objeto, la persona o el hecho, así como las relaciones que se establecen entre los distintos símbolos se abstraen una vez el usuario ha establecido contacto con el concepto con múltiples ejemplos y en múltiples situaciones, por lo que MAREMA nos permite descubrir qué relaciones tiene establecidas con mayor importancia y cuáles no suele entrenar.

Debido al marco social en el que nos movemos, y la multitud de problemáticas asociadas al envejecimiento, la institucionalización, fallecimiento de personas de nuestro entorno u otros factores propios del paso del tiempo, las emociones más recurrentes en su comunicación interna y externa se caracterizan por tener un contenido desagradable, provocando una cadena relacional de recuerdos conexionados por la misma temática emocional.

La aplicación de MAREMA nos ayuda a trabajar las áreas cognitiva, emocional y conductual de manera transversal.

A nivel cognitivo, nos permite construir ideas y trabajar la memoria a corto, medio y largo plazo, orientarlos a la realidad y a la creatividad propia de la generación de ideas de futuro, así como el desempeño de habilidades cognitivas necesarias para la elaboración de pensamiento complejo y la mejora de la atención al momento presente. 
A nivel emocional, nos ayuda a ampliar el número de emociones experimentadas en la cotidianidad, así como a aceptar y a validar lo que surge sin juzgar, fomentando el autoconocimiento emocional de su historia vivida y la variabilidad de las emociones en diferentes momentos y circunstancias, trabajando la fluctuación como algo positivo, facilitando de este modo la defusión y el distanciamiento de sus eventos privados, (emociones, ideas, pensamientos, sensaciones físicas...)

A nivel conductual, entendiendo que el pensamiento es conducta, utilizar MAREMA nos Ileva a trabajar de un modo muy amplio, provocando activación conductual más allá de la aplicación del instrumento.

MAREMA es un instrumento que trabaja de manera transversal todos los marcos relacionales. La aplicación de MAREMA está orientada al trabajo flexible, por lo que tanto el contenido de las preguntas como el número de participantes puede ser variado, el instrumento también podría ser utilizado de manera individual como valoración sobre deterioro cognitivo (línea de trabajo en vías de desarrollo), observando el nivel que pueden llegar a contestar de manera coherente 0 valorando de forma subjetiva que relación emocional tienen establecida, por un discurso recurrente sobre los mismos sucesos, o si su corriente de pensamiento se centra únicamente en el pasado. Pudiendo así elaborar distintas preguntas o centrarnos en el trabajo de los niveles que queremos reforzar para evitar la pérdida de habilidades cognitivas, para elaborar un recuerdo de su historia de vida más amplio.

Los niveles son 13, los niveles del 1 al 6 pueden ser trabajados también con juegos o ejercicios que permitan realizar una conexión relacional tangible, los niveles del 7 al 13 son

relaciones analógicas, que requieren que las anteriores no estén deterioradas para poder trabajarse.

Podemos crear una batería de preguntas que se adapten a la población o nivel cultural de las personas con las que estamos trabajando, para ello en cada nivel tenemos que idear una pregunta que obligue a establecer la relación a trabajar para poder ser respondida.

La temporalidad tiene el objetivo de establecer una flexibilidad psicológica en la que podamos manejar el contenido mental a través de toda la vida actual, pasada o futura.

Las preguntas centradas en el futuro deben ser formuladas para evocar a la creatividad y el distanciamiento de su propia realidad provocando un pensamiento global que permita pensar en otras personas, más allá de lo que ya existe, lo establecido o de sí mismos.

Las preguntas basadas en el presente, tienen que buscar orientar a la realidad, focalizar la atención en lo que está sucediendo, ha sucedido o puede suceder, durante el día en el que se realizan las preguntas, de este modo es más probable lograr una activación conductual proactiva del participante.

Las preguntas del pasado tienen el objetivo de trasladar mentalmente al sujeto a través de su historia vital de manera activa y no dirigida por un pensamiento recurrente, tomando así el control de lo que hacen con su mente en lugar de reaccionar ante la emoción o pensamiento.

El pasado lejano, tiene que estar dirigido a la evocación de recuerdos del inicio de la vida o primeras experiencias, cuando aún no se habían producido hitos vitales que marcasen o anclasen emocionalmente su recuerdo del pasado, obviando muchas otras vivencias que no son recordadas por no establecerse mayor número de relaciones respecto a ellas que en las consideradas "vitales" en la historia de vida del que lo narra.

Realizar preguntas de MAREMA de manera aleatoria facilitara que todos los participantes se sientan involucrados en la actividad en los grupos heterogéneos, respondiendo así a las necesidades de los profesionales que trabajan en instituciones en la que encontramos muchas variables en las características, gustos o necesidades de los/las residentes. 


\section{LA ACTUACIÓN DEL PROFESIONAL DE LA PSICOLOGÍA EN LAS RESIDENCIAS DE PERSONAS MAYORES}

Tabla 6. MAREMA. Instrumento para el entrenamiento de Marcos Relacionales de manera transversal a través del tiempo en mayores.

\begin{tabular}{|c|c|c|c|c|}
\hline Marco Relacional & Futuro & Presente & Pasado & Pasado Lejano \\
\hline $\begin{array}{l}\text { - IGUALDAD (“es”, “es cómo”, } \\
\text { "va con", "es igual”) }\end{array}$ & $\begin{array}{c}\text { ¿Qué cosas crees } \\
\text { que no cambiarán } \\
\text { nunca? }\end{array}$ & $\begin{array}{l}\text { ¿Quién va vestido } \\
\text { del mismo color } \\
\text { que tú? }\end{array}$ & $\begin{array}{l}\text { ¿Qué persona } \\
\text { disfrutaba de algún } \\
\text { programa de } \\
\text { televisión contigo } \\
\text { hace } 20 \text { años? }\end{array}$ & $\begin{array}{l}\text { ¿Qué personas } \\
\text { recuerdas que } \\
\text { naciesen el mismo } \\
\text { año que tú? }\end{array}$ \\
\hline $\begin{array}{l}\text { - DISTINCIÓN("es distinto", "es } \\
\text { diferente") }\end{array}$ & $\begin{array}{l}\text { ¿Qué cosas } \\
\text { comerán, que } \\
\text { ahora no } \\
\text { comemos? }\end{array}$ & $\begin{array}{l}\text { ¿Qué diferencias } \\
\text { hay entre vivir } \\
\text { donde vives ahora } \\
\text { o dónde vivías } \\
\text { antes? }\end{array}$ & $\begin{array}{c}\text { ¿Qué cosas hacían } \\
\text { las mujeres distintas } \\
\text { de los hombres hace } \\
40 \text { años? }\end{array}$ & $\begin{array}{l}\text { ¿Qué diferencias } \\
\text { había en tu infancia } \\
\text { entre el pueblo y la } \\
\text { ciudad? }\end{array}$ \\
\hline Marco Relacional & Futuro & Presente & Pasado & Pasado Lejano \\
\hline $\begin{array}{l}\text { - ESPACIALES (arriba-abajo, } \\
\text { cerca-lejos, lleno-vacío, encima- } \\
\text { debajo, etc.) }\end{array}$ & $\begin{array}{l}\text { ¿Para qué usarías } \\
\text { las azoteas de los } \\
\text { edificios? }\end{array}$ & $\begin{array}{l}\text { ¿Cómo es la } \\
\text { lámpara que } \\
\text { tenemos arriba? }\end{array}$ & $\begin{array}{l}\text { Descríbeme la parte } \\
\text { de arriba De la } \\
\text { Iglesia de tu pueblo }\end{array}$ & $\begin{array}{l}\text { ¿Cuando eras niño } \\
\text { podías tocar el } \\
\text { fondo del río? }\end{array}$ \\
\hline $\begin{array}{l}\text { - OPOSICIÓN("es opuesto", "es } \\
\text { lo contrario") }\end{array}$ & $\begin{array}{c}\text { ¿Qué cosas } \\
\text { cambiaran por } \\
\text { completo dentro de } \\
100 \text { años? }\end{array}$ & $\begin{array}{l}\text { ¿Qué programa de } \\
\text { televisión te gusta } \\
\text { más y cuál menos? }\end{array}$ & $\begin{array}{l}\text { ¿Con quién discutías } \\
\text { más por tus ideas de } \\
\text { joven? }\end{array}$ & $\begin{array}{l}\text { ¿Cuál era tu comida } \\
\text { favorita de niño y } \\
\text { cuál odiabas? }\end{array}$ \\
\hline $\begin{array}{l}\text { COMPARACIÓN("es mayor } \\
\text { que", "es menor que", "más", } \\
\text { "menos", "más alto") }\end{array}$ & $\begin{array}{c}\text { ¿Qué transporte } \\
\text { inventarán para ir } \\
\text { más rápido? }\end{array}$ & $\begin{array}{c}\text { ¿Quién es más alto } \\
\text { de los que están } \\
\text { aquí? }\end{array}$ & $\begin{array}{l}\text { ¿Qué hermano tardó } \\
\text { más en irse de casa? }\end{array}$ & $\begin{array}{l}\text { ¿Quién era el } \\
\text { pequeño de tus } \\
\text { vecinos? }\end{array}$ \\
\hline $\begin{array}{l}\text { DEÍCTICOS(“yo-tú”, "aquí- } \\
\text { allí", "antes-después”) }\end{array}$ & $\begin{array}{c}\text { ¿Qué crees que } \\
\text { haríamos si } \\
\text { viviésemos en la } \\
\text { luna en unos años? }\end{array}$ & $\begin{array}{c}\text { ¿Qué ropa llevas } \\
\text { ahora y cuál te va a } \\
\text { poner después? }\end{array}$ & $\begin{array}{l}\text { ¿Dónde estabas tú } \\
\text { hace } 30 \text { años, y } \\
\text { dónde estaba yo? }\end{array}$ & $\begin{array}{l}\text { ¿Qué diferencia } \\
\text { había antes y } \\
\text { después de la } \\
\text { guerra? }\end{array}$ \\
\hline $\begin{array}{l}\text { - COORDINACIÓN (cuando dos } \\
\text { elementos o más pertenecen a un } \\
\text { mismo conjunto, por ejemplo: } \\
\text { barbo, sardina). }\end{array}$ & $\begin{array}{l}\text { ¿Qué tendrán en } \\
\text { común un ruso y } \\
\text { un español en diez } \\
\text { años? }\end{array}$ & $\begin{array}{l}\text { Menciona dos } \\
\text { personas que te } \\
\text { parezcan } \\
\text { inteligentes }\end{array}$ & $\begin{array}{l}\text { Las personas que } \\
\text { iban a misa cuando } \\
\text { tenías } 30 \text { años, ¿qué } \\
\text { tenían en común? }\end{array}$ & $\begin{array}{l}\text { Cuando eras niño, } \\
\text { ¿qué especies había } \\
\text { en el río? }\end{array}$ \\
\hline $\begin{array}{l}\text { - SUBORDINACIÓN(un } \\
\text { elemento pertenece a un } \\
\text { conjunto, por ejemplo: perro- } \\
\text { mamífero). }\end{array}$ & $\begin{array}{c}\text { ¿Qué ideas } \\
\text { políticas crees que } \\
\text { tendrán tus nietos? }\end{array}$ & $\begin{array}{l}\text { ¿Qué equipo de } \\
\text { fútbol te gusta } \\
\text { más? }\end{array}$ & $\begin{array}{l}\text { ¿Qué nacionalidad } \\
\text { tienen tus hijos? }\end{array}$ & $\begin{array}{l}\text { ¿En qué provincia } \\
\text { nació tu madre? }\end{array}$ \\
\hline
\end{tabular}




\begin{tabular}{|c|c|c|c|c|}
\hline $\begin{array}{l}\text { PARTE A TODO (un elemento } \\
\text { es parte de, por ejemplo: pétalo } \\
\text { parte de una flor). }\end{array}$ & $\begin{array}{l}\text { ¿Qué piezas crees } \\
\text { que formarán los } \\
\text { coches del futuro? }\end{array}$ & $\begin{array}{c}\text { ¿Qué ingredientes } \\
\text { llevaba lo que has } \\
\text { comido hoy? }\end{array}$ & $\begin{array}{c}\text { ¿Qué partes tenía la } \\
\text { casa donde vivías } \\
\text { antes? }\end{array}$ & $\begin{array}{c}\text { ¿Qué ingredientes } \\
\text { tenía el pan cuando } \\
\text { eras niño? }\end{array}$ \\
\hline $\begin{array}{l}\text { SUPRAORDENACIÓN (un } \\
\text { conjunto integra al elemento. por } \\
\text { ejemplo: ovíparo integra a las } \\
\text { gallinas). }\end{array}$ & $\begin{array}{l}\text { ¿Qué personas } \\
\text { crees que dejaran } \\
\text { de comer animales } \\
\text { en unos años? }\end{array}$ & $\begin{array}{c}\text { ¿Qué personas } \\
\text { conoces que sean } \\
\text { cristianas? }\end{array}$ & $\begin{array}{c}\text { ¿Qué personas de tu } \\
\text { juventud se fueron } \\
\text { del país? }\end{array}$ & $\begin{array}{l}\text { ¿Qué niños iban al } \\
\text { colegio contigo? }\end{array}$ \\
\hline $\begin{array}{l}\text { TODO A PARTE(un elemento } \\
\text { se compone de, por ejemplo: } \\
\text { Agua contiene oxígeno, una } \\
\text { tortilla contiene huevos). }\end{array}$ & $\begin{array}{l}\text { ¿Quién crees que } \\
\text { formará parte de tu } \\
\text { familia dentro de } \\
10 \text { años? }\end{array}$ & $\begin{array}{l}\text { ¿Quién compone } \\
\text { ahora tu familia? }\end{array}$ & $\begin{array}{c}\text { ¿Quién formaba } \\
\text { parte de tu familia } \\
\text { hace } 35 \text { años? }\end{array}$ & $\begin{array}{l}\text { ¿Quién formaba } \\
\text { parte de tu familia } \\
\text { cuando eras niño? }\end{array}$ \\
\hline $\begin{array}{l}\text { - CAUSA-EFECTO (un elemento } \\
\text { es causa de otro, por ejemplo: } \\
\text { Enfriar el agua la convierte en } \\
\text { hielo). }\end{array}$ & $\begin{array}{l}\text { Teniendo en } \\
\text { cuenta el tiempo } \\
\text { este año ¿qué } \\
\text { cosecha crees que } \\
\text { tendremos? }\end{array}$ & $\begin{array}{c}\text { ¿Quién ha sonreído } \\
\text { hoy gracias a ti? }\end{array}$ & $\begin{array}{c}\text { ¿Alguien sufrió por } \\
\text { algo que tú hicieses } \\
\text { de joven? }\end{array}$ & $\begin{array}{l}\text { ¿Cómo ganaste tu } \\
\text { primer sueldo? }\end{array}$ \\
\hline Marco Relacional & Futuro & Presente & Pasado & Pasado Lejano \\
\hline $\begin{array}{l}\text { - PROXIMIDAD(un elemento } \\
\text { suele estar cerca del otro, en el } \\
\text { espacio o en el tiempo, pero sin } \\
\text { relación de inclusión } \\
\text { causalidad entre ellos, por } \\
\text { ejemplo: primavera-lluvia, } \\
\text { ciencia-tecnología.). }\end{array}$ & $\begin{array}{l}\text { Cuando ya no estés } \\
\text { aquí, ¿cómo crees } \\
\text { que se } \\
\text { transportarán los } \\
\text { humanos? }\end{array}$ & $\begin{array}{l}\text { ¿Con qué persona } \\
\text { de tu entorno casi } \\
\text { no has hablado? }\end{array}$ & $\begin{array}{l}\text { Cuando te casaste, } \\
\text { ¿quién gobernaba el } \\
\text { país? }\end{array}$ & $\begin{array}{c}\text { ¿En qué ocupabas el } \\
\text { tiempo libre la } \\
\text { primera vez que } \\
\text { pudiste votar? }\end{array}$ \\
\hline
\end{tabular}

\section{CONCLUSIONES}

La idea de realizar este trabajo surge de encontrarnos con la ausencia de regulación y la falta del establecimiento formal bajo unos criterios unificados, por parte de los organismos correspondientes, de cuáles son las funciones -y las líneas de actuación- del profesional de la psicología en las residencias de personas mayores, lo cual ocasiona degradación de la profesión así como innumerables casos de intrusismo profesional. De ahí que se plantee esta propuesta.

Vemos que la actuación del profesional de la Psicología en los centros residenciales para personas mayores se realiza, en general, como parte del equipo interdisciplinar. Pensamos que esto se debe a que se han reconocido sus competencias para la coordinación con el resto de profesionales. Sin embargo, al no estar sus funciones incluidas en las normativas y convenios establecidos de manera oficial, su aplicación es arbitraria en muchas ocasiones.

Por otro lado, para llevar a cabo de modo eficiente y eficaz la intervención del profesional de la psicología sería idóneo establecer la ratio. La propuesta que planteamos es que sea una ratio de 50 residentes por profesional de la psicología. De este modo creemos que queda cubierta de una manera adecuada la atención psicológica de residentes y familias. 


\section{LA ACTUACIÓN DEL PROFESIONAL DE LA PSICOLOGíA EN LAS RESIDENCIAS DE PERSONAS MAYORES}

En cuanto a la intervención dirigida a residentes nos encontramos con que el profesional de la Psicología, como parte del equipo interdisciplinar, tiene que participar en la elaboración e implementación del PAI. Dentro del mismo, este profesional, realiza la planificación del programa terapéutico dirigido a la rehabilitación y mantenimiento de capacidades cognitivas y funcionales, la prevención y tratamiento de problemas emocionales, modificación de conductas disfuncionales, favorecer el proceso de adaptación e integración al centro, ofrecer atención psicoterapéutica individual, elaborar los programas de intervención cognitiva, emocional y conductual, etc.), pues si se omite la parte psicológica del enfoque bio-psico- social ya no se trata de una atención integral y de calidad.

En cuanto a la intervención dirigida a las familias nos encontramos con que esta se centra en el acompañamiento, asesoramiento y apoyo a los familiares de referencia. Es importante que el psicólogo-a ayude a redefinir el nuevo rol que adopta la familia tras el ingreso. También ofrece información sobre el usuario-a, media en posibles conflictos familia-centro 0 entre las familias, participa en acciones formativas, coordina grupos terapéuticos y de ayuda mutua, etc.

En cuanto a la intervención dirigida a la institución, vemos que el/la psicólogo-a persigue aportar calidad en la labor asistencial y favorecer un buen clima de trabajo paliando el efecto de burnout, los conflictos laborales, etc. Pero también ofrecer apoyo formativo en cuestiones de salud, envejecimiento, trabajo en equipo, etc. Además, participa en la elaboración e implementación de planes de buenas prácticas y prevención del maltrato. $Y$ hay que reconocer que es importante en la optimización de las vías de intercomunicación institucional.

\section{BIBLIOGRAFÍA}

Arenas i Guarch, S. y Pratginestós Gonzalo, E. (2006) El Papel del Psicólogo en los Centros Residenciales Geriátricos. Col-legi Oficial de Psicòlegs de Catalunya

Colegio Oficial de Psicólogos (1998) Perfiles del psicólogo http://www.cop.es/perfiles

Consejería de Bienestar Social del Principado de Asturias. La familia, contigo. http://www.acpgerontologia.com/documentacion/lafamiliacontigo.pdf

Díaz Veiga, P. Márquez-González, M. Sancho Castiello, M. Yanguas Lezaun, J(2009). Cómo nos ayudan las emociones a envejecer bien. Obra Social Caixa Catalunya.

Hayes, S.C.; Barnes-Holmes, D. y Roche, B. (2001). Relational frame theory: A post- Skinnerian account of human language and cognition. New York: Kluwer Academic/Plenum.

López Pérez, B.; Fernández-Pinto, I. y Márquez-González, M. (2008). Educación emocional en adultos y personas mayores. Revista Electrónica de Investigación Psicoeducativa. № 15, Vol 6, pp:501-522.

Mielgo Casado, A.; Ortiz Muños, MD; Ramos Noesí, C. (2001) El rol del psicólogo que trabaja con personas mayores. Definición y desarrollo profesional. Intervención Psicosocial, Vol. $10,{ }^{\circ} 3$ - Págs. 395-409.

Pérez Álvarez, M. Universidad de Oviedo. La Activación Conductual y la Desmedicalización de la Depresión. Papeles del Psicólogo. 2007. Vol.28 (2).pp. 97 - 110

Soldevilla A. Ribes R. Filella G.Agulló MJ(2005) Objetivos y contenidos de un programa de educación emocional para personas mayores. Emociona t. Revista Iberoamericana de educación (ISSN:1681-5653) 